$\underline{\text { Preprint typeset in JHEP style - PAPER VERSION }}$

UTTG-12-01, QMUL-PH-03-06

\title{
Finite Number of States, de Sitter Space and Quantum Groups at Roots of Unity
}

\author{
Philippe Pouliot \\ on leave from \\ Physics Department \\ Physics Department \\ University of Texas at Austin \\ E) Queen Mary, University of London \\ London, E1 4NS, UK \\ Austin, TX 78712 USA \\ p.pouliot@qmul.ac.uk
}

\begin{abstract}
This paper explores the use of a deformation by a root of unity as a tool to build models with a finite number of states for applications to quantum gravity. The initial motivation for this work was cosmological breaking of supersymmetry. We explain why the project was unsuccessful. What is left are some observations on supersymmetry for q-bosons, an analogy between black holes in de Sitter and properties of quantum groups, and an observation on a noncommutative quantum mechanics model with two degrees of freedom, depending on one parameter. When this parameter is positive, the spectrum has a finite number of states; when it is negative or zero, the spectrum has an infinite number of states. This exhibits a desirable feature of quantum physics in de Sitter space, albeit in a very simple, non-gravitational context.
\end{abstract}

KEywords: Finite number of states, cosmological constant, de Sitter space, black holes, quantum groups at roots of unity, noncommutative quantum mechanics, cosmological breaking of supersymmetry. 


\section{Contents}

1. Introduction $\quad 1$

2. The $q$-deformed oscillator $\quad 3$

2.1 Review of the $q$-boson algebra for $q$ real 3

2.2 Review of the $q$-boson algebra for $q$ a root of unity 4

2.3 Supersymmetry of the $q$-oscillator at an odd root of unity 7

2.4 More supersymmetric examples 9

2.5 Supersymmetry breaking $\quad 11$

3. Using quantum groups for black holes in de Sitter 13

3.1 Review of $S U(2)_{q} \quad 13$

3.1.1 The representations of $U_{q}(S U(2)) \quad 14$

$\begin{array}{lll}3.1 .2 & \text { Tensor products of representations } & 15\end{array}$

3.2 Black holes in de Sitter and $S U(2)_{q} \quad 16$

3.3 The thermodynamics for $S U(2)_{q}$ particles, versus $S U(2) \quad 17$

$\begin{array}{lll}3.3 .1 & \text { At low temperature } & 18\end{array}$

$\begin{array}{lll}3.3 .2 & \text { At high temperature } & 18\end{array}$

4. A toy model for de Sitter space (without quantum groups) 18

4.1 The model of Bellucci, Nersessian and Sochichiu 19

$4.2 q$-deformation with 2 degrees of freedom 20

4.3 Noncommutative SUSY quantum mechanics with a magnetic field 21

5. Conclusion 22

A. Mathematica Code 23

\section{Introduction}

We know very little about quantum gravity. Perhaps the most important insight about what a theory of quantum theory of gravity ought to look like is the holographic principle [1]. This already suggests a drastic reduction in the number of degrees of 
freedom required for a theory of quantum gravity, compared to what we have in a quantum field theory ${ }^{1}$. The observation by astronomers that the expansion of the Universe is accelerating could be explained by a non-zero dark energy or cosmological constant and that we live in an asymptotically de Sitter space-time. According to Fischler [2], to Banks [3], to Bousso [4], and to others, this suggests that a further, even more drastic, reduction is required. Namely, that only a finite number of quantum states is sufficient to describe all the causal physics that happens within one observer's cosmological horizon.

This would indicate that there is something fundamentally wrong with the quantum field theories and string theories that we usually work with: they all have an infinite number of states.

Furthermore, a cosmological constant necessarily breaks supersymmetry, and Banks conjectured [3] that the scale of supersymmetry breaking is directly tied to the size of the cosmological constant. Note however that the scale associated with supersymmetry breaking $m_{S U S Y}$ is much larger than that set by the cosmological constant $\Lambda$. Thus, supersymmetry has to be broken by a "large" amount if the origin of supersymmetry breaking is the cosmological constant. Phenomenologically, a relation such as $m_{S U S Y} \propto \Lambda^{1 / 8}$ would work.

In this paper, we are looking for toy models that would support this conjecture. There are of course many ways to truncate theories to a finite number of states. The main tool that we will be using is deforming by a root of unity the simple harmonic oscillators that are ubiquitous in perturbation theory. In this way, we readily obtain theories with a finite number of states. However, this procedure does not necessarily break supersymmetry. We have not found an example where supersymmetry is broken by a "large" amount, but we have not ruled out their existence either. Thus our approach has been unsuccessful so far. Also, the idea of using quantum groups as a discretization tool is not new, see for example [5] or [6]. We hope however that our presentation is new, emphasizing the simplicity of using $q$-bosons and quantum groups.

In summary, in section 2 , we review the properties of $q$-bosons, and discuss their supersymmetric properties. In section 3, we review $S U(2)_{q}$ and its thermodynamics properties and draw a caricature of how it could be applied to black hole physics. In section 4, we review the non-commutative quantum mechanics model of Bellucci, Nersessian and Sochichiu and supersymmetrize it.

\footnotetext{
${ }^{1}$ This is particularly true in loop quantum gravity, where quantum groups at roots of unity and finite/discrete number of states naturally enter the formalism. See [7] and, for an extensive list of references to the original literature, the excellent recent article [8].
} 


\section{The $q$-deformed oscillator}

\subsection{Review of the $q$-boson algebra for $q$ real}

Although perhaps not as familiar as the quantum group $S U(2)_{q}$, we will begin with $q$-bosons. This is a simpler starting point, since there is only one degree of freedom. In addition, all quantum groups can be built out of $q$-bosons. Please see the excellent book [9] for further details and an extensive list of references to the original literature.

There is an essential distinction between $q$ real and $q$ a root of unity. Although our true interest is in the case where $q$ is a root of unity, let us discuss first the simpler case when $q$ is a positive real number. When $q \in \mathbb{R}^{+}$, the representations of the $q$-boson algebra are rather similar to the ordinary boson. When $q$ is real, there is an infinite number of states and when $q$ is a root of unity, there will be a finite number of states.

The $q$-boson algebra for $q$ real consists of the creation and annihilation operators $a_{+}$and $a_{-}$, and the number operator $N^{2}$. They satisfy the defining relations:

$$
a_{-} a_{+}-q a_{+} a_{-}=q^{-N}, \quad\left[N, a_{ \pm}\right]= \pm a_{ \pm} .
$$

The last relation can also be written as $q^{N} a_{ \pm} q^{-N}=q^{ \pm 1} a_{ \pm}$. We see that these relations interpolate between boson $(q=1)$ and fermion $(q=-1)$ commutation relations, which also makes it clear that working out the details of the $q$-fermion is easy (see Hayashi in [10]). There are of course many equivalent ways of writing equation 2.1. One common one is $A_{+}=a_{+} q^{N / 2}, A_{-}=q^{N / 2} a_{-}$for which the first relation in 2.1 becomes $A_{-} A_{+}-q^{2} A_{+} A_{-}=1^{3}$.

This algebra is known as the $q$-Heisenberg-Weyl algebra $U_{q}\left(h_{4}\right)$ but we will call it here the $q$-boson algebra for short. For doing quantum mechanics, one needs a hermitian conjugation (an involution) †; here it is simply

$$
a_{-}^{\dagger}=a_{+}, \quad a_{+}^{\dagger}=a_{-}, \quad N^{\dagger}=N .
$$

The notion of a $q$-integer will be useful:

$$
[x]=\frac{q^{x}-q^{-x}}{q-q^{-1}} .
$$

\footnotetext{
${ }^{2}$ Our conventions differ from [9] by $q^{1 / 2} \rightarrow q, a \rightarrow a_{+}$and $\bar{a} \rightarrow a_{-}$.

${ }^{3}$ They are not quite equivalent, as explained in [12], where three different deformations are considered when $q$ is real. The main physical distinction between the cases is that the operators of position and momentum can be bounded or unbounded. When unbounded, these operators turn out to not be self-adjoint but to have a self-adjoint extension. This is the case for equation 2.1, which is the second case considered in [12]. In all cases, for $q$ real, their spectrum is continuous. For $q$ a root of unity, the spectrum is finite and therefore the issue does not arise.
} 
The algebra 2.1 has a central element: $q^{-N}\left([N]-a_{+} a_{-}\right)$. The actual algebra we are interested in is equations 2.1 modded out by setting this central element to zero [11]. The result is that there is only one unitary irreducible representation of this restricted algebra, and it is infinite dimensional. It is the one we would expect, closely related to that of the ordinary boson. This representation can be realized by a Fock space, with a vacuum state $|0\rangle$ that is annihilated by $a_{-}$, and

$$
a_{+}|n\rangle=\frac{a_{+}^{n}}{\sqrt{[n] !}}|0\rangle, \quad N|n\rangle=n|n\rangle,
$$

with the $q$-factorial defined recursively by $[n] !=[n]([n-1] !)$. In the restricted algebra, the first relation in 2.1 is actually equivalent to the two relations

$$
a_{+} a_{-}=[N], \quad a_{-} a_{+}=[N+1]
$$

The position and momentum operators are defined in the usual way:

$$
X=\frac{a_{+}+a_{-}}{\sqrt{2}} \quad \text { and } \quad P=\frac{-a_{+}+a_{-}}{i \sqrt{2}} .
$$

They obey the commutation relation

$$
[X, P]=i([N+1]-[N])
$$

In physical terms, this means that Planck's constant is no longer a constant, but depends on the excitation energy of the system, and actually diverges at very high energy. Readers are referred to Biedenharn in [10] for other such statements on the physics of $q$-bosons.

For the hamiltonian of a $q$-deformed oscillator, we make the natural choice

$$
H=\frac{1}{2}\left(a_{+} a_{-}+a_{-} a_{+}\right)=\frac{1}{2}([N]+[N+1])
$$

Its spectrum for small values of $n$ is almost evenly spaced, and thus very similar to the ordinary simple harmonic oscillator (see first two columns of figure 3 ). For large values of $n$ however, for $q<1,[n] \rightarrow q^{-n}$ and grows exponentially fast and the spectra become very different.

\subsection{Review of the $q$-boson algebra for $q$ a root of unity}

Let us now describe the $q$-boson for $q$ a root of unity. Our presentation will be elementary and our focus is on illustrating the finiteness of the number of states. The algebra 
consists of the creation and annihilation operators $a_{+}$and $a_{-}$, and of $L$. The defining relations are [9] (page 190):

$$
a_{-} a_{+}-q a_{+} a_{-}=L^{-1} \quad \text { and } \quad L a_{ \pm} L^{-1}=q^{ \pm 1} a_{ \pm}
$$

for $q=e^{2 \pi i / k}$ a root of unity. The number operator $N$, such that $L=q^{N}$, is not welldefined: it is multi-valued and periodic; however, operators such as $[N]$ are well-defined and can be expressed in terms of $L$ and $q$. It follows from 2.9 that

$$
a_{+} a_{-}=\frac{L-L^{-1}}{q-q^{-1}}=[N] \quad \text { and } \quad a_{-} a_{+}=\frac{q L-q^{-1} L^{-1}}{q-q^{-1}}=[N+1] \text {. }
$$

We have $[x]=\frac{q^{x}-q^{-x}}{q-q^{-1}}=\frac{\sin 2 \pi x / k}{\sin 2 \pi / k}$, so that now there are identities that follow from properties of the sine function: $\left[x+\frac{k}{2}\right]=-[x],[x+k]=[x],\left[\frac{k}{2}\right]=0$. With these relations, one can show [9] by induction that $a_{-} a_{+}^{n}=q^{n} a_{+}^{n} a_{-}+[n] a_{+}^{n-1} L^{-1}$. Specializing to $k$ odd for now, $a_{ \pm}^{k}$ commutes with $a_{ \pm}$and $L$. Because an operator like $a_{+} a_{-}$can take both positive and negative values, one has to define the hermitian conjugation $\dagger$ as follows in order to fix up the signs: $a_{+}^{\dagger}=a_{-} \epsilon([N]), a_{-}^{\dagger}=\epsilon([N]) a_{+}$, and $L^{\dagger}=L^{-1}$, where $\epsilon$ is a sign function: $\epsilon(x)=\left\{\begin{aligned} 1, & \text { if } x \geq 0, \\ -1, & \text { if } x<0 .\end{aligned}\right.$

We can now review the representation theory [9]. For $q$ an odd root of unity, it is much more complicated than for $q$ real. There are nilpotent, cyclic, semi-cyclic and indecomposable representations. However, only one of them is interesting to us.

We are interested in the representation for which $a_{ \pm}^{k}=0$; hence it is called nilpotent. It is unitary and irreducible. Label its carrier space by $|n\rangle, n=0,1, \ldots, k-1$. Then the generators act by:

$$
\begin{aligned}
L|n\rangle & =q^{n}|n\rangle, \quad a_{+}|k-1\rangle=0, \quad a_{-}|0\rangle=0 \\
a_{+}|n\rangle & =\sqrt{|[n+1]|}|n+1\rangle, \quad n \neq k-1, \\
a_{-}|n\rangle & =\epsilon([n]) \sqrt{|[n]|}|n-1\rangle, \quad n \neq 0 .
\end{aligned}
$$

Thus it can be built by acting in the usual way with creation operators: $|n\rangle=\frac{\left(a_{+}\right)^{n}}{\sqrt{|[n]| !}}|0\rangle$, with $a_{-}|0\rangle=0$ and $|k\rangle=0$, and || denotes the absolute value. So the representation has a finite number of states, $k^{4}$.

\footnotetext{
${ }^{4}$ For $k$ even, there are two nilpotent representations, with $a_{ \pm}^{k / 2}=0$, and each with $k / 2$ states.
} 
There are more representations, those for which $a_{ \pm}^{k} \neq 0$. We can simply ignore them for our purposes ${ }^{5}$.

Now a few words about the properties of the position and momentum operators. We can define the manifestly hermitian coordinates ${ }^{6}$

$$
X=\frac{1}{\sqrt{2}}\left(a_{+}+a_{+}^{\dagger}\right) \quad \text { and } \quad P=\frac{1}{i \sqrt{2}}\left(a_{+}-a_{+}^{\dagger}\right) .
$$

They satisfy

$$
[X, P]=i(|[N+1]|-|[N]|) .
$$

We have studied the spectrum numerically. In figure 1 , we can find the discrete, finite, spectrum of $X$ for $q=e^{2 \pi i / 25}$. The total length of the line grows like $k^{1 / 2}$. $X=0$ is always an eigenvalue, and the spectrum is symmetric with respect to $X \rightarrow$ $-X$. Except close to the origin, we see that two eigenvalues reside at each value of $X$, with a very narrow splitting of order $e^{-c k}$, where $c$ is independent of $k$ but grows larger for eigenvalues farther from the origin. Finally, such pairs of very nearly degenerate eigenvalues are separated from their next neighbors by a distance that shrinks like $c^{\prime} k^{-1 / 2}+\mathcal{O}\left(k^{-3 / 2}\right)$ where $c^{\prime}$ is another constant. Thus, we see that a $q$-deformation is a subjectively nice discretization of the real line, in the sense that even for relatively small $k$, the line is chopped into $k$ bits all of much the same length.

\footnotetext{
${ }^{5}$ In a continuum quantum field theory, these representations would be solitons. They are characterized by a continuous parameter $\phi$, defined modulo $2 \pi$, analogous to an (additive) magnetic charge. In quantum field theory, because of unitarity, they would necessarily arise. They would be created non-perturbatively in pairs of "charge" $\phi$ and $-\phi$ of total charge zero, and ignoring them would violate unitarity. For our discretized theories with a finite number of states, the rules are unitary without these solitons. For completeness, we will review now these cyclic and semicyclic representations, for odd $k$. They are labelled by $l \in \mathbb{C}^{*}, \mu, \nu \in \mathbb{C}$. The action on the carrier space $|n\rangle, n=0,1, \ldots, k-1$ is

$$
\begin{aligned}
L|n\rangle & =l q^{n}|n\rangle, \quad a_{+}|k-1\rangle=\mu|0\rangle, \quad a_{-}|0\rangle=\nu|k-1\rangle \\
a_{+}|n\rangle & =|n+1\rangle, \quad n \neq k-1, \\
a_{-}|n\rangle & =\left(l^{-1}[n]+q^{n} \mu \nu\right)|n-1\rangle, \quad n \neq 0,
\end{aligned}
$$

and the values of the Casimirs are $L^{k}=l^{k}, a_{+}^{k}=\mu, a_{-}^{k}=\nu \prod_{n=1}^{k-1}\left(l^{-1}[n]+q^{n} \mu \nu\right)$, related by $a_{-}^{k} a_{+}^{k}=$ $[N+1][N+2] \cdots[N+k]$. On the restricted algebra, defining an angle $l=e^{i \phi},-\pi \leq \phi<\pi$, there is a further relation $\mu \nu=\frac{l-l^{-1}}{q-q^{-1}}=[\phi]$ and the action of $a_{-}$simplifies to $a_{-}|n\rangle=\left(\frac{q^{n} l-q^{-n} l^{-1}}{q-q^{-1}}\right)|n-1\rangle$.

${ }^{6}$ We could also have used the hermitian operator $\tilde{X}=\frac{1}{\sqrt{2}}\left(a_{-}+a_{-}^{\dagger}\right)\left(\right.$ or $\left.\tilde{P}=\frac{1}{i \sqrt{2}}\left(a_{-}+a_{-}^{\dagger}\right)\right) . \tilde{X}$ almost commutes with $X$ (only 2 entries are non-zero in the matrix of the commutator $[X, \tilde{X}]$ ), and their eigenvalues are the same. However, $\tilde{X}$ does not have a simple commutation relation with $P$ ).
} 


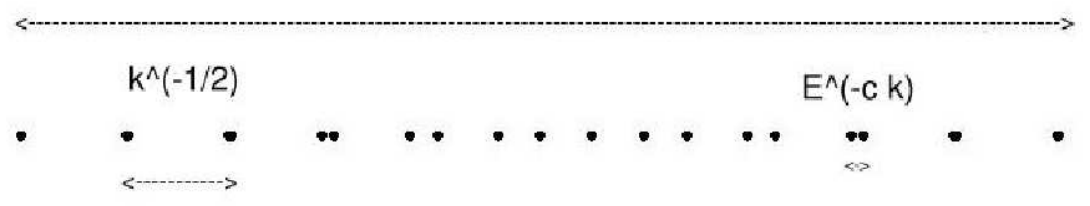

Figure 1. The discretization of the spectrum of $X$.

\subsection{Supersymmetry of the $q$-oscillator at an odd root of unity}

In this section, we make a very simple, but strange, observation: the ordinary, nonsupersymmetric, simple harmonic oscillator can lead to a supersymmetric model after $q$-deformation by an odd root of unity.

There are at least 3 hermitian hamiltonians one can consider, that reduce to the usual s.h.o. in the limit $q \rightarrow 1^{7}$ :

$$
\begin{aligned}
& H_{A}=\frac{1}{2}\left(a_{+} a_{-}+a_{-} a_{+}\right), \quad H_{B}=\frac{1}{2}\left|a_{+} a_{-}+a_{-} a_{+}\right|, \text {and } \\
& H_{C}=\frac{1}{2}\left(\left|a_{-} a_{+}\right|+\left|a_{+} a_{-}\right|\right)=\frac{1}{2}\left(a_{+}^{\dagger} a_{+}+a_{-}^{\dagger} a_{-}\right)=\frac{1}{2}(|[N+1]|+|[N]|) .
\end{aligned}
$$

Their spectra, which consist of $k$ states, are compared in figure 2, along with the undeformed s.h.o. The maximum energy is $E_{\max }=\frac{1}{\sin \left(\frac{2 \pi}{k}\right)} \rightarrow \frac{k}{2 \pi}$, and it grows linearly at large $k$ for all three hamiltonians.

The spectrum of hamiltonian $H_{A}$ has both negative and positive energies, and is symmetric with respect to the zero energy point. It is like a Dirac sea (despite there being no fermions here.) The spectrum of $H_{C}$ has doubly degenerate states, except for one state of energy very close to the two ground states. This state has energy $\frac{1}{2 \cos \frac{\pi}{k}} \rightarrow \frac{1}{2}+\frac{\pi^{2}}{4 k^{2}}$. As $k \rightarrow \infty, H_{C}$ has three ground states of energy $1 / 2$.

\footnotetext{
${ }^{7}$ In the case where $k$ is an even root of unity, the hamiltonians $H_{A}, H_{B}$ and $H_{C}$ coincide and the spectrum is positive and exactly doubly degenerate.
} 


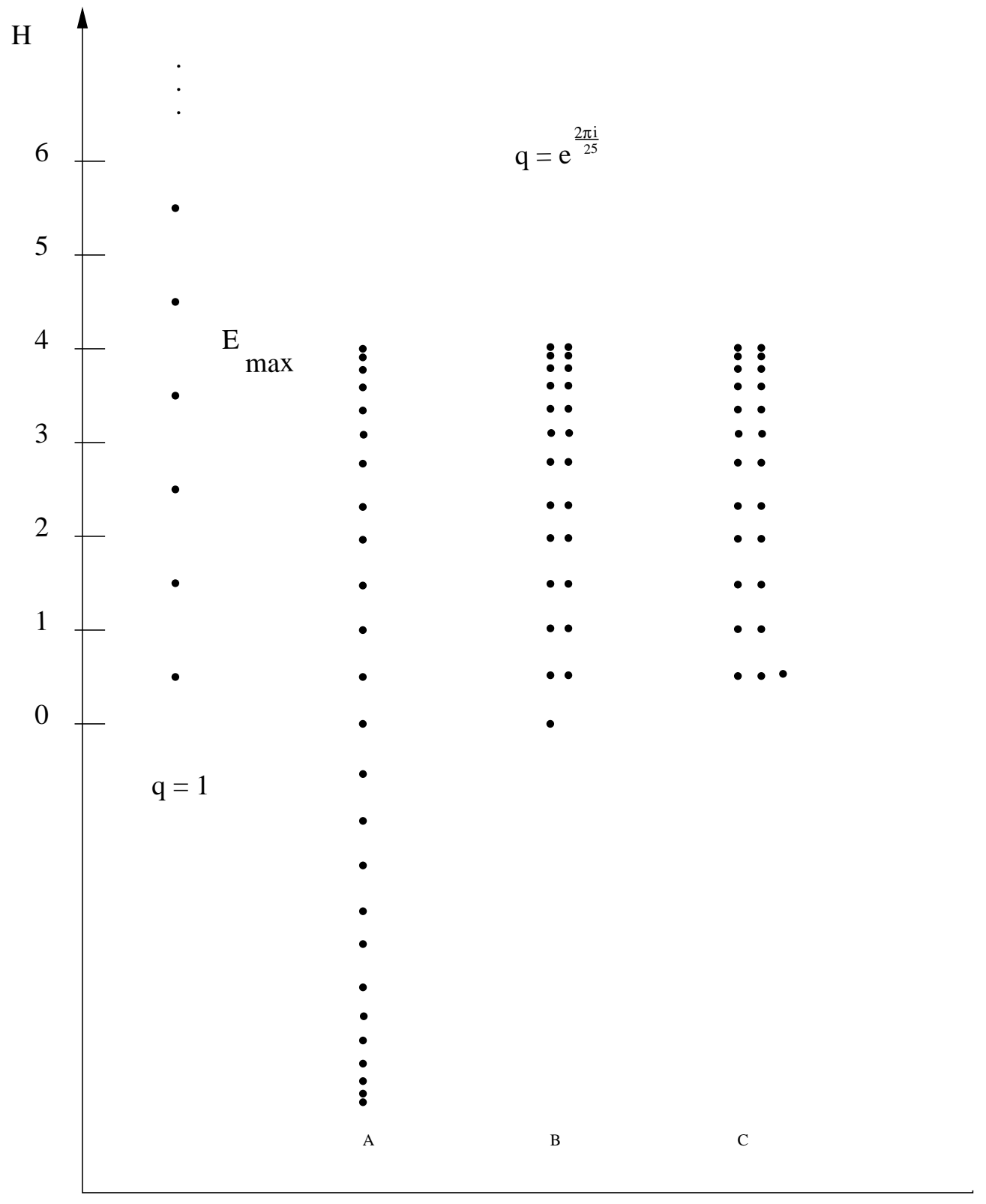

Figure 2. The spectra of the ordinary s.h.o. along with $H_{A}, H_{B}$ and $H_{C}$.

The spectrum of $H_{B}$ is supersymmetric. This is rather strange: never have we mentioned fermions, yet this hamiltonian has a single zero energy ground state, and the rest of its spectrum is doubly degenerate. For concreteness, one can write down 
two hermitian supercharges:

$$
Q_{1}=R \Sigma_{1} \quad \text { and } \quad Q_{2}=R \Sigma_{2}
$$

where

$$
\Sigma_{1}=\left(._{1} .^{1^{1}} \cdot{ }^{\cdot 1}\right), \quad \Sigma_{2}=\left(._{i} \cdot{ }^{0{ }^{-i}}{ }^{\cdot{ }^{-i}}\right)
$$

and $R$ is the diagonal matrix of entries $\frac{1}{\sqrt{2}} \sqrt{|[N+1]+[N]|}$. They satisfy $\left\{Q_{\alpha}, Q_{\beta}\right\}=$ $2 \delta_{\alpha \beta} H_{B}$ for $\alpha, \beta=1,2$. We hope that this $q$-boson with hamiltonian $H_{B}$ could be an interesting way to introduce fermions and supersymmetry in some applications.

\subsection{More supersymmetric examples}

When $q$ is real $\in(0,1)$, a straightforward way to supersymmetrize the $q$-deformed oscillator is by introducing two ordinary fermions and two hermitian conjugate supercharges:

$$
Q_{+}=\sqrt{2}\left(a_{-} \psi_{+}\right), \quad \text { and } \quad Q_{-}=\sqrt{2}\left(a_{+} \psi_{-}\right)
$$

with $\left\{\psi_{ \pm}, \psi_{ \pm}\right\}=1,\left\{\psi_{ \pm}, \psi_{\mp}\right\}=0, \psi_{ \pm}^{\dagger}=\psi_{\mp}$ and $a_{ \pm}^{\dagger}=a_{\mp}$. The hamiltonian is:

$$
H=\frac{1}{2}\left\{Q_{+}, Q_{-}\right\}=[N]+([N+1]-[N]) \psi_{+} \psi_{-}
$$

Its spectrum is shown in the last column of Figure 3. 


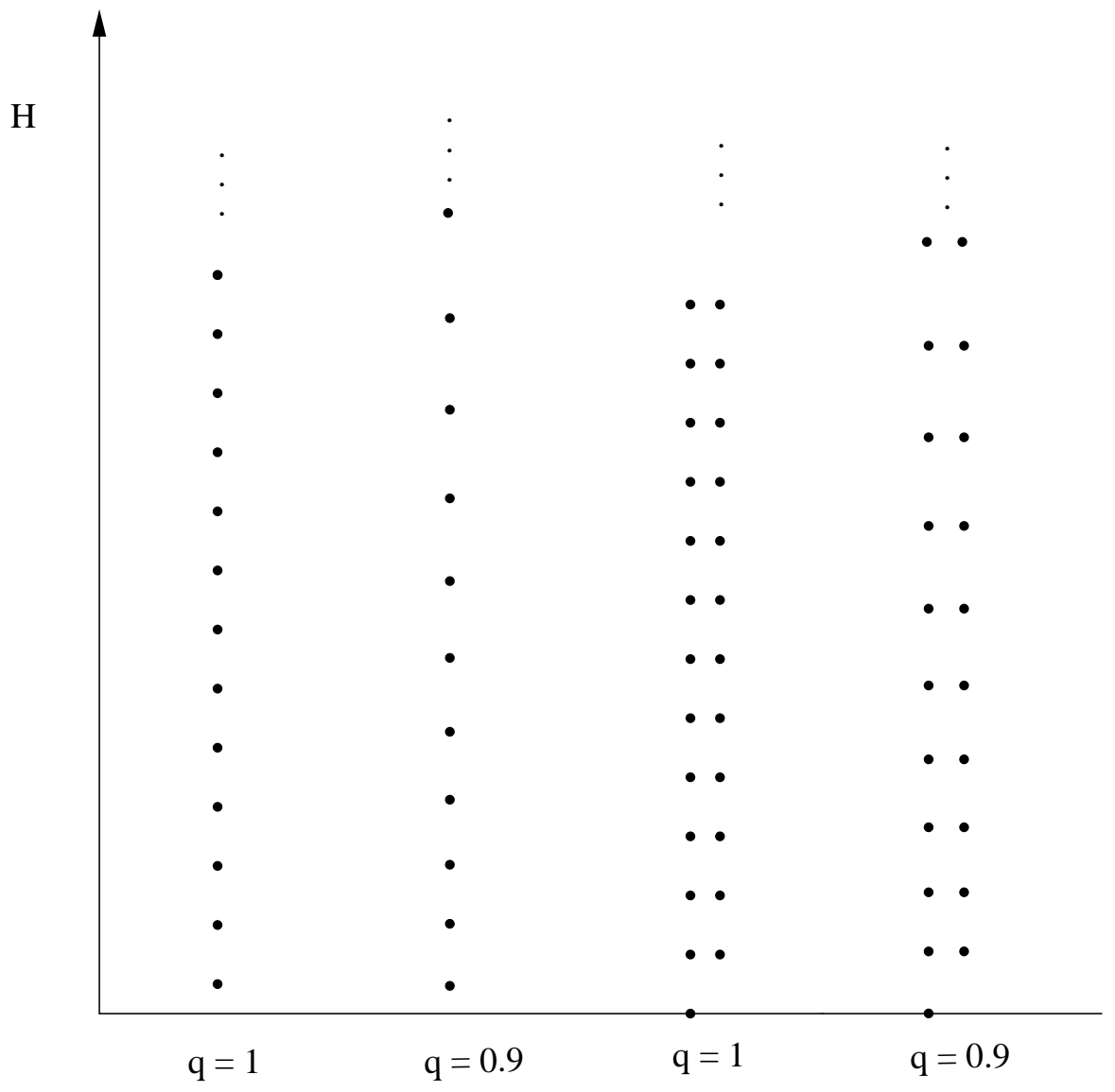

Figure 3. In column 4, the spectrum of the $q$-deformed SUSY h.o. for $q=0.9$.

For $q$ a root of unity, these supercharges do not lead to a hermitian hamiltonian, so a little more work is required. Introducing

$$
Q_{+}=\sqrt{2}\left(a_{-} \psi_{+}\right), \quad \text { and } \quad Q_{-}=\sqrt{2}\left(\epsilon([N]) a_{+} \psi_{-}\right),
$$

we get a supersymmetric hermitian hamiltonian

$$
H=\frac{1}{2}\left\{Q_{+}, Q_{-}\right\}=|[N]|+(|[N+1]|-|[N]|) \psi_{+} \psi_{-},
$$

with two zero energy ground states and whose non-zero spectrum is quadruply degenerate (Figure 4). There are four supercharges. Again, there is a finite number of states, with $E_{\max } \rightarrow k / 2 \pi$. 


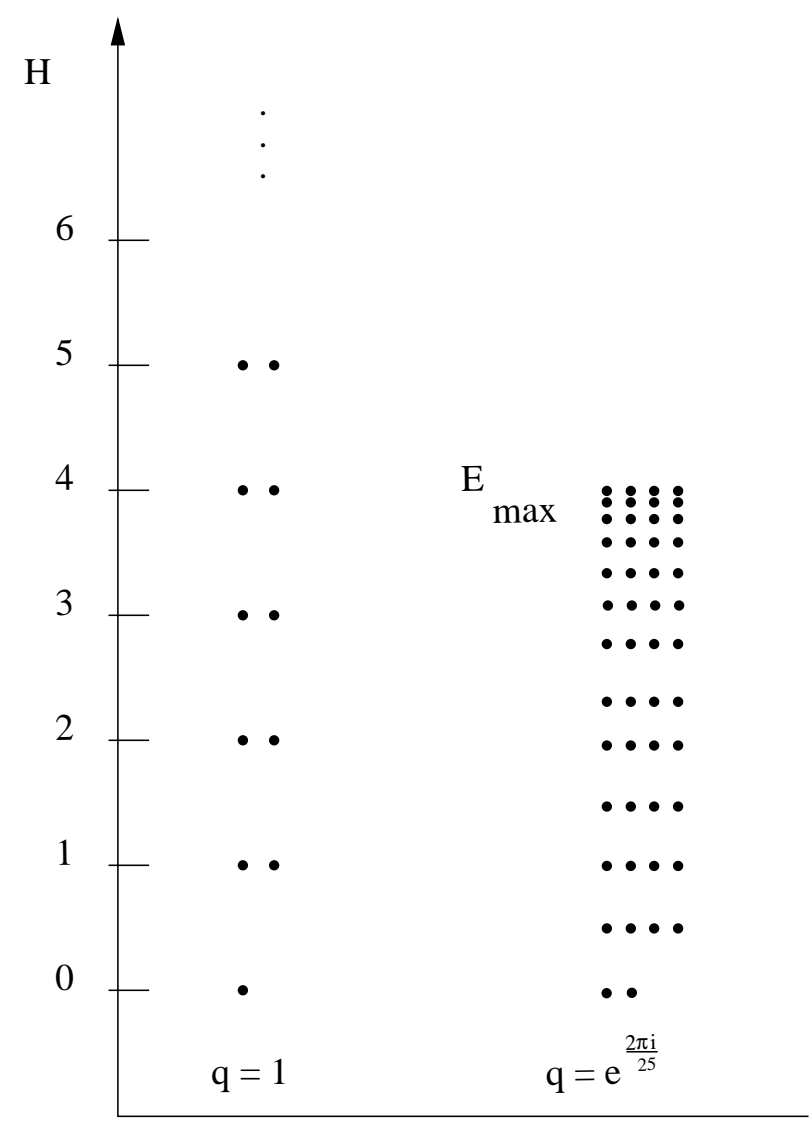

Figure 4. The spectra of the $q$-deformed SUSY s.h.o. for $q=e^{2 \pi i / 25}$.

\subsection{Supersymmetry breaking}

Example 1:

Let us now consider adding a quartic hermitian potential to the supersymmetric hamiltonian $H_{B}$ of earlier.

$$
H=H_{B}+\frac{\lambda}{16} X^{4} .
$$

This hamiltonian is not supersymmetric. The spectrum shows the small splittings one would expect (Figure 5). The ground state energy is different from zero by a quantity of order $\lambda$ and the energy splittings of the higher energy states are also of order $\lambda$. 


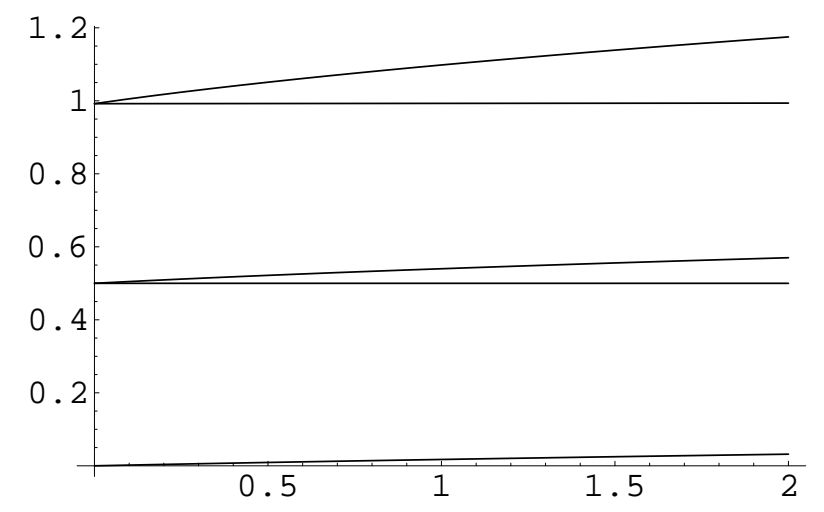

Figure 5. Splitting of eigenvalues by $\lambda X^{4}$ potential.

Example 2:

Consider a one-dimensional supersymmetric quantum mechanics model [15] with a supercharge $Q=P \psi_{1}+W(X) \psi_{2}$, and commutation relations $[X, P]=i$, and $\left\{\psi_{i}, \psi_{j}\right\}=$ $\delta_{i j}$. The hamiltonian is

$$
H=\frac{1}{2}\{Q, Q\}=\frac{P^{2}}{2}+\frac{W^{2}}{2}+i W^{\prime} \psi_{1} \psi_{2}
$$

We would like to consider a badly behaved superpotential, for example $W=g / X$. Then $H=\frac{1}{2}\left(P^{2}+g^{2} / X^{2}+g \sigma_{3} / X^{2}\right)$. For an odd root of unity, the matrix $X$ is not invertible ( 0 is an eigenvalue.) Thus let's deform by an even root of unity, $q=e^{i \pi / k}$. Consider the representation with $k$ states built as $\frac{a_{+}^{n}}{\sqrt{|[n]|}}|0\rangle$ for which $X$ does not have a zero eigenvalue. The matrix $X$ is now invertible. We then simulated the system on a classical computer. The spectrum has a double degeneracy, which is irrelevant here.

\begin{tabular}{r|ll|l}
$k$ & $E_{0}$ & $E_{1}$ & $E_{1}-E_{0}$ \\
\hline & \multicolumn{3}{|l}{} \\
40 & 0.404 & 0.696 & 0.292 \\
80 & 0.202 & 0.348 & 0.146 \\
160 & 0.101 & 0.174 & 0.073 \\
320 & 0.0504 & 0.0868 & 0.0364 \\
640 & 0.0252 & 0.0434 & 0.0182
\end{tabular}

Table 1: lowest two energy levels for $W=3 / X$. 
In table 1, we have tabulated the numerical values of the lowest two energy levels of this system, for different values of $k$ and the specific case of the superpotential $W=3 / X$. It is quite clear that the model is becoming supersymmetric in the limit $k \rightarrow \infty$. Supersymmetry is however broken for any finite value of $k$. It is broken by a "small" amount though, as it is quite apparent that the ground state energy $E_{0}$ goes to zero as $1 / k$ and that the energy splittings $E_{1}-E_{0}$ also go to zero as $1 / k$. For cosmological breaking of supersymmetry, one would like the energy splittings to decrease slower, at a fractional power of $k$ for example.

\section{Using quantum groups for black holes in de Sitter}

We started this project hoping that $S U(2)_{q}$ representations would exhibit properties qualitatively similar to those of black holes in de Sitter space. The key point is that black holes in de Sitter space cannot be larger than the size of the cosmological horizon. This raises a question: suppose one takes a black hole which is almost the size of the cosmological horizon, and throws in it some extra matter. What happens? We will naively assume that we can have such matter at our disposal and that the matter can be thrown into the black hole while keeping the size of the cosmological horizon fixed. Then one scenario is that such a black hole could behave like a quantum group. Namely, as we throw in more and more matter, the black hole gets smaller and smaller, and its energy decreases. We will illustrate how $S U(2)_{q}$ at a root of unity has this curious feature. After a review of the fairly well-known properties of $S U(2)_{q}$, we will give our results on using $S U(2)_{q}$ for black holes.

\subsection{Review of $S U(2)_{q}$}

The reader familiar with $S U(2)_{q}$ would like to skip this section. Quantum groups have several applications in theoretical physics, such as the quantum inverse scattering method and integrable models, conformal field theory in two dimensions, nuclear physics and non-commutative geometry. For an introduction addressed to physicists, see again Biedenharn's book [9].

Now we will review some well-known facts about $S U(2)_{q}$. This quantum group is by definition the universal enveloping algebra generated by $J_{ \pm}$and $K$ subject to the commutation relations:

$$
K J_{ \pm} K^{-1}=q^{ \pm 2} J_{ \pm} \quad\left[J_{+}, J_{-}\right]=\frac{K-K^{-1}}{q-q^{-1}}=\left[2 J_{3}\right]
$$

Here $q=e^{2 \pi i / k}$, and $k$ is a positive odd number (the case with $k$ even is slightly more complicated). As $q \rightarrow 1$, these commutation relations reduce to those of the Lie algebra 
of $S U(2)$, for $K=q^{2 J_{3}}$. The hermiticity operation acts as $K^{\dagger}=K^{-1}$ and $J_{ \pm}^{\dagger}=J_{\mp}$. The generalization of the Casimir invariant of $S U(2)$ is:

$$
C=J_{-} J_{+}+\left[J_{3}+\frac{1}{2}\right]^{2}-\left[\frac{1}{2}\right]^{2} .
$$

One can easily check that $C$ commutes with $J_{ \pm}$and $K$ by using the identities $[x+y]=$ $q^{x}[y]+[x] q^{-y}$ and $[x][y-z]+[y][z-x]+[z][x-y]=0$. We will use this Casimir invariant as our hamiltonian (the constant $[1 / 2]^{2}$ subtracted to make the ground state energy vanish). As we will see below, there are many representations of this algebra, and most of them can be ignored for our purposes. These representations are labelled by three more Casimir invariants, $J_{+}^{k}, J_{-}^{k}, K^{k}$. They are not fully independent, as there is one algebraic relation between the 4 invariants.

\subsubsection{The representations of $U_{q}(S U(2))$}

When $q$ is real, the representations of $U_{q}(S U(2))$ are a continuous deformation of the representations of $S U(2)$. When $q$ is a root of unity, the situation is drastically different.

(A) The closest representations to those of $S U(2)$ arise when $J_{ \pm}^{k}=0$. Then we have unitary irreducible representations for angular momentum $j=0,1 / 2,1, \ldots, j_{\text {max }}$ up to a maximum value of $j_{\max }=\frac{k}{2}-1$. These representations have $2 j+1$ states, and are continuous deformations of the usual representations of $S U(2)$. The representation with $j=\frac{k-1}{2}$ is not unitary, but still irreducible. Then, there is a family of representations of dimension $2 k$. These representations are both non-unitary and reducible but not fully reducible (this is called indecomposable). These non-unitary representations are quite undesirable physically. We will be cavalierly dropping them, but see [13] for the rational behind this statement.

(B) When $J_{ \pm}^{k} \neq 0$, there are cyclic representations, of dimension $k$ and $2 k$, labelled by families of continuous parameters. Some of them are unitary and others are not. These representations form a separate ring, i.e. separate from the $J_{ \pm}^{k}=0$ representations, and we can also ignore them for our purposes, as we did for the cyclic representations of $q$-bosons. In physical terms, the eigenvalues of $J_{ \pm}^{k}$ label different solitonic superselection sectors, and we declare that we work only in the $J_{ \pm}^{k}=0$ sector.

Therefore, the only physical representations are $j=0,1 / 2, \ldots, j_{\max }$ of $(\mathrm{A})$ and, what is most essential for us, there is a finite number of them.

Returning to the Casimir, evaluated on the unitary representations $j=0, \ldots, j_{\text {max }}$ :

$$
C=\frac{\sin ^{2} \frac{\pi(2 j+1)}{k}-\sin ^{2} \frac{\pi}{k}}{\sin ^{2} \frac{2 \pi}{k}}=\frac{1}{2 \sin ^{2} \frac{2 \pi}{k}}\left(\cos \frac{2 \pi}{k}-\cos \frac{2 \pi(2 j+1)}{k}\right) .
$$


There is also a notion of $q$-dimension $D$ of a representation:

$$
D=[2 j+1]=\frac{\sin \frac{2 \pi(2 j+1)}{k}}{\sin \frac{2 \pi}{k}} .
$$

For example, if $k=5$, the $j=0,1 / 2,1,3 / 2$ representations are unitary; $j=0,3 / 2$ have $q$-dimension 1 and Casimir $0 ; j=1 / 2,1$ have $q$-dimension $\frac{1-\sqrt{5}}{2}$ and Casimir $-1+\sqrt{5}$. But the 5 -dimensional $j=2$ is not unitary, its $q$-dimension is zero. The $j=5 / 2$ representation is part of the 10 -dimensional " $9 / 2=5 / 2+2$ " indecomposable representation, also with $q$-dimension 0 .

A key feature of the spectrum as given by the Casimir is a "hump", which becomes more apparent for larger values of $k$. Since $C$ is given by a trigonometric functions, we see that $C(j)$ starts at zero, increases up to a maximum value $j_{\text {top }}=\frac{k-3}{4}$ at the "top of the hill", where it takes a maximum value of $C_{t o p} \approx \frac{k^{2}}{4 \pi^{2}}$ for large $k$, and then decreases back to zero.

\subsubsection{Tensor products of representations}

The formula we will be using for decomposing the tensor product of the unitary representations is quite simple [9]:

$$
\left(j_{1} \otimes j_{2}\right)=\left(\bigoplus_{j=\left|j_{1}-j_{2}\right|}^{\min \left(j_{1}+j_{2}, k-j_{1}-j_{2}-2\right)} j\right) .
$$

This formula is obtained by throwing away the non-unitary or indecomposable representations, as justified in [13] (see section 5 on page 528) ${ }^{8}$.

${ }^{8}$ For completeness, we will give the formulas including these representations [14]. First, when we tensor two unitary representations of spin small enough, the decomposition is analogous to $S U(2)$ :

$$
\left.\left(j_{1} \otimes j_{2}\right)\right|_{j_{1}+j_{2}<\frac{k-1}{2}}=\bigoplus_{j=\left|j_{1}-j_{2}\right|}^{j_{1}+j_{2}} j .
$$

When the sum of the spins of the two unitary representations we multiply is larger than $\frac{k-1}{2}$, then non-unitary/indecomposable representations occur in the decomposition:

$$
\begin{aligned}
\left.\left(j_{1} \otimes j_{2}\right)\right|_{2\left(j_{1}+j_{2}\right) \text { even }, \geq k-1} & =\left(\bigoplus_{j=\left|j_{1}-j_{2}\right|}^{\min \left(j_{1}+j_{2}, k-j_{1}-j_{2}-2\right)} j\right) \oplus \frac{k-1}{2} \oplus\left(m_{j_{1}, j_{2}} \oplus \frac{2 k-1}{2}\right) \\
\left.\left(j_{1} \otimes j_{2}\right)\right|_{2\left(j_{1}+j_{2}\right) \text { odd }, \geq k-1} & =\left(\bigoplus_{j=\left|j_{1}-j_{2}\right|}^{\min \left(j_{1}+j_{2}, k-j_{1}-j_{2}-2\right)} j\right) \oplus\left(m_{j_{1}, j_{2}} \oplus \frac{2 k-1}{2}\right),
\end{aligned}
$$

where $m_{j_{1}, j_{2}}$ is the known number of times that the various $k-\frac{1}{2}$ representations occur, which can be worked out by equating the total dimensions on both sides. 
Let's give two examples of the use of the formula: for a small number like $k=5$ and a larger number like $k=103$. For $k=5$, the tensor product of two spins $\frac{1}{2}$ is as usual: $1 / 2 \otimes 1 / 2=0+1$, but other tensor products are truncated: $1 / 2 \otimes 3 / 2=1$, $1 \otimes 1=0+1,1 \otimes 3 / 2=1 / 2,3 / 2 \otimes 3 / 2=0$.

Let's see the pattern for these tensor decompositions with $k=103$. The unitary reps are $j=0,1 / 2,1, \ldots, 50, \frac{101}{2}$. Then there is the non-unitary rep $j=51$ and the indecomposable rep $j=\frac{205}{2}$. The top of the hill is at $j_{\text {top }}=25$. For simplicity, let's just look at tensor products where $j$ is an integer. We get for the product of a small representation times one near the top of the hill: $1 \otimes 24=23 \oplus 24 \oplus 25$; for two very large representations: $50 \otimes 50=0 \oplus 1$; for the tensor product of many small representations: $1^{\otimes 50}=50 \oplus \mu 49 \oplus \cdots$, where the multiplicity of the 49 appearing in the decomposition is $\mu=49$; etc.

\subsection{Black holes in de Sitter and $S U(2)_{q}$}

Now, we want to use $S U(2)_{q}$ to make an analogy with black holes in de Sitter. Choosing a value of $k$ is like choosing the dimension of the Hilbert space and the size of the cosmological horizon, and so will be very large. An elementary particle will be a representation with $j$ small. A black hole will be a "medium-size" unitary representation, with $j$ smaller than but close to $j_{\text {top }}=\frac{k-3}{4}$. The energy, i.e. the size, of black holes grows like $j$, until it abruptly stops growing when the maximum energy/size has been reached. The "large" representations with $j$ larger than $j_{\text {top }}$ will form a "hidden sector", because as we saw from the tensor product decompositions, they are hard to reach from the point of view of the fundamental particles in the sense that it takes of order $k$ tensor products of fundamental particles to reach them. It maybe be that the universe started in a state constituting mostly of small $j$ particles. For $k$ large, it may be that the time scale for populating the hidden sector is very large compared to the present age of the universe.

Now, when we "drop" a fundamental particle into a black hole, we use the tensor product decomposition to find the result of the interaction. What we get are black hole states, some of them conventional with $j<j_{\text {top }}$, but others have $j>j_{\text {top }}$ and thus have lower energy: dropping a particle into the black hole has had the effect of "shrinking" some of the black hole states, lowering their energy, and putting them into the "hidden sector".

When two black holes interact, states with all values of $j$ are produced. When two states from the hidden sector interact, they collapse mostly into fundamental particles. Clearly, one would need a theory of the dynamics to make sense of these interactions.

In summary, the merit of this caricature of de Sitter space is that black holes have a maximal size and energy. When more matter is dropped into such a black hole, it 
manages to shrink and lower its energy.

\subsection{The thermodynamics for $S U(2)_{q}$ particles, versus $S U(2)$}

Continuing the analogy, we would like to see if the natural thermodynamics properties of $S U(2)_{q}$ will be useful to describe black holes. The total number of black hole states is of order $k^{2}$, much larger than the number of fundamental particles, of order 1 . For the real world, we would like a much faster, exponential growth. Can this be obtained from quantum groups? We will not address this question in this paper, but only begin the discussion by looking at the high and low temperature properties of the partition function of $S U(2)_{q}$.

To calculate thermodynamics properties, we should decide what we are going to do with the states in the "hidden sector". One possibility is to simply include them in the partition function ${ }^{9}$.

For the Casimir $C$ as the hamiltonian, with $\beta=1 / T$ the inverse temperature, the partition function is:

$$
Z_{S U(2)_{q}}=\sum_{m=1}^{k-1} m e^{-\frac{\beta}{2 \sin ^{2} \frac{2 \pi}{k}}\left(\cos \frac{2 \pi}{k}-\cos \frac{2 \pi m}{k}\right)} .
$$

It can be evaluated in close form:

$$
\begin{array}{r}
Z_{S U(2)_{q}}=k \sum_{m=1}^{k-1} e^{-\frac{\beta}{2 \sin ^{2} \frac{2 \pi}{k}}\left(\cos \frac{2 \pi}{k}-\cos \frac{2 \pi m}{k}\right)} \\
=k e^{-\gamma \cos \frac{2 \pi}{k}}\left(e^{-\gamma}+k I_{0}(\gamma)\right)
\end{array}
$$

where $\gamma=\frac{\beta}{2 \sin ^{2} \frac{2 \pi}{k}}$ and $I_{0}$ is a Bessel function. For undeformed $S U(2)$, with the Hamiltonian $H=j(j+1)$, the partition function is:

$$
Z_{S U(2)}=\sum_{n=0}^{\infty}(n+1) e^{-\beta n(n+2) / 4} .
$$

The usual thermodynamics quantities are the free energy $F$ defined by $Z=e^{-\beta F}$, the entropy $S=\beta^{2} d F / d \beta$, the average energy $E=d(\beta F) / d \beta$ with $E=F+T S$, and the specific heat $c_{v}=d E / d T$.

\footnotetext{
${ }^{9}$ Another possibility, if the temperature is low enough, is to ignore the hidden sector states and work with a system in quasi-equilibrium. At low temperature then, rather trivially, the partition function for $S U(2)_{q}$ has the same leading behavior as the one for $S U(2)$, except that the temperature is shifted: $Z \rightarrow 1+2 e^{-\frac{3 \beta}{4}\left(1-\frac{1}{3} \tan ^{2}\left(\frac{\pi}{k}\right)\right)}$, that is $\beta \rightarrow \beta\left(1-\frac{1}{3} \tan ^{2}\left(\frac{\pi}{k}\right)\right)$, which goes over to the $S U(2)$ partition function for large $k$. At high temperature, we should certainly not ignore the hidden sector.
} 


\subsubsection{At low temperature}

For $S U(2)$ at low temperature, the leading contribution to the partition function of course comes from the lowest energy states, so we have $Z \rightarrow 1+2 e^{-3 \beta / 4}, F \rightarrow \frac{-2}{\beta} e^{-3 \beta / 4}$, $S \rightarrow(2+3 \beta / 2) e^{-3 \beta / 4}, E \rightarrow \frac{3}{2} e^{-3 \beta / 4}$ and $c_{v} \rightarrow \frac{9 \beta^{2}}{8} e^{-3 \beta / 4}$. The specific heat is of course very small at low temperature.

For $S U(2)_{q}$, we have $Z \rightarrow k\left(1+e^{-\beta\left(1-\frac{1}{4} \cos ^{-2} \frac{\pi}{k}\right)}\right)$. This partition function does not at all reduce to the one for $S U(2)$ in the $k \rightarrow \infty$ limit. This is because of the low energy states from the hidden sector. Instead, as $k \rightarrow \infty$, we have $Z \rightarrow k+k e^{-3 \beta / 4}$, from which we get $F \rightarrow \frac{-1}{\beta}\left(\log k+e^{-3 \beta / 4}\right), E \rightarrow \frac{3}{4} e^{-3 \beta / 4}, S \rightarrow \log k+(1+3 \beta / 4) e^{-3 \beta / 4}$ and $c_{v} \rightarrow \frac{9 \beta^{2}}{16} e^{-3 \beta / 4}$.

\subsubsection{At high temperature}

The partition function of $S U(2)$ at high temperature goes like $Z_{S U(2)} \rightarrow \frac{2}{\beta}$. The free energy is negative and goes as $\frac{1}{\beta} \log \frac{\beta}{2}$, characteristic of one quantum mechanical degree of freedom. The entropy is $S=-\log \frac{\beta}{2}+1$, the energy $E=\frac{1}{\beta}$ and $c_{v}=1$.

At high temperature and large $k$, the partition function for $S U(2)_{q}$ becomes $Z \rightarrow$ $k^{2}\left(1-\frac{\beta k^{2}}{8 \pi^{2}}\right)$, valid when $\beta k^{2}$ is small. From that we get: $F=-\frac{\log k^{2}}{\beta}+\frac{k^{2}}{8 \pi^{2}}$. The free energy and entropy are characteristic of a quantum mechanical system with $k^{2}$ states. $E=\frac{k^{2}}{8 \pi^{2}} ; S=\log k^{2}$ and $c_{v}=0$. We see that raising the temperature does not further increase the entropy. The average energy is half the maximum energy level: further increasing the temperature does not increase the average energy and we never reach $E_{\max }$

Clearly, the high temperature properties of $S U(2)_{q}$ are typical of a system with a finite number of states. There is a maximum average energy, a maximum entropy and the specific heat goes to zero. These are desirable features for the thermodynamics of de Sitter space.

\section{A toy model for de Sitter space (without quantum groups)}

In this section, we will describe a simple quantum mechanical model with only two degrees of freedom that very roughly features a property that we expect a complete quantum theory of gravity to possess: that there is a finite number of states for a positive cosmological constant, and an infinite number of states for a negative one.

The authors of [17] made the following very interesting observation. A noncommutative quantum mechanics model on the infinite plane, with a magnetic field, with two degrees of freedom, has a conserved angular momentum $J$. The model has a parameter, let us call it $\Lambda$ in analogy with the cosmological constant. When this parameter 
is negative or zero, there is an infinite number of quantum states, as one would expect for anti-de Sitter space and Minkowski space. When the parameter is positive, there is a finite number of states, as one would expect for de Sitter space, as long as one considers only the sector with a given value of the conserved total angular momentum.

In section 4.1, we will review the model of [17]. In section 4.2, we look at what happens to the BNS model if we deform it by a root of unity. In section 4.3, we supersymmetrize the BNS model. We looked at breaking supersymmetry by a large amount, but did not find a way to do it.

\subsection{The model of Bellucci, Nersessian and Sochichiu}

The model [17] is a 2-dimensional noncommutative quantum mechanics system with a magnetic field. The hamiltonian is $H=\frac{p^{2}}{2}+\frac{\omega^{2} x^{2}}{2}$, with the commutation relations $\left[x_{1}, x_{2}\right]=i \theta,\left[x_{i}, p_{j}\right]=i \delta_{i j}$ and $\left[p_{1}, p_{2}\right]=i B$, and $\theta>0$. Since the hamiltonian is quadratic, it can be diagonalized exactly by a Bogolyubov transformation [16]. The result is

$$
H_{1}=\frac{\omega_{+}}{2}\left(\beta_{+} \beta_{-}+\beta_{-} \beta_{+}\right)+\frac{\omega_{-}}{2}\left(\alpha_{+} \alpha_{-}+\alpha_{-} \alpha_{+}\right)
$$

with $\left[\alpha_{-}, \alpha_{+}\right]=1,\left[\beta_{-}, \beta_{+}\right]=\operatorname{sgn}(\Lambda)$ and $[\alpha, \beta]=0$. Thus the spectrum is the product of two decoupled harmonic oscillators with different frequencies, $H=\omega_{+}\left(n_{+}+\frac{1}{2}\right)+$ $\omega_{-}\left(n_{-}+\frac{1}{2}\right)$, with $n_{ \pm}=0,1,2, \ldots$ and

$$
\omega_{ \pm}=\frac{1}{2}\left|B+\omega^{2} \theta \pm \sqrt{\left(B-\omega^{2} \theta\right)^{2}+4 \omega^{2}}\right|=\frac{1}{4 \theta}\left|\mathcal{E}+\Lambda \pm \sqrt{(\mathcal{E}-\Lambda)^{2}+4 \Lambda}\right|
$$

for $\mathcal{E}=1+\omega^{2} \theta^{2}$. The physics is quite different depending on the sign of $\Lambda=\theta B-1$. There is a conserved angular momentum $j=n_{+}+\operatorname{sgn}(\Lambda) n_{-}$. At fixed values of the angular momentum, if $\Lambda$ is negative, there is an infinite number of states allowed, but if $\Lambda$ is positive, there is only a finite number of states.

The transformation required to get to this hamiltonian depends on the sign of $\Lambda$ [17]. At a first stage, one goes from the variables $(x, p)$ to an intermediate set $(a, b)$. At this stage the hamiltonian takes the form:

$$
H_{2}=\frac{|\Lambda|}{2 \theta}\left(b_{+} b_{-}+b_{-} b_{+}\right)-\frac{i \sqrt{|\Lambda|}}{\theta}\left(b_{+} a_{-}-a_{+} b_{-}\right)+\frac{\mathcal{E}}{2 \theta}\left(a_{+} a_{-}+a_{-} a_{+}\right),
$$

where $a_{ \pm}=\frac{x_{1} \mp i x_{2}}{\sqrt{2 \theta}}, b_{ \pm}=\sqrt{\frac{\theta}{2|\Lambda|}}\left(\pi_{1} \mp i \pi_{2}\right)$ and $\pi_{i}=p_{i}-\epsilon_{i j} x_{j} / \theta$.

At the second stage, then for $\Lambda<0$, the operators $\alpha$ and $\beta$ are related to $a$ and $b$ by the further substitutions: $\left(\begin{array}{c}a_{+} \\ b_{+}\end{array}\right)=U\left(\begin{array}{c}\alpha_{+} \\ \beta_{+}\end{array}\right)$, with $U=\left(\begin{array}{c}\phi \cosh \chi \phi \sinh \chi \\ \bar{\phi} \sinh \chi \bar{\phi} \cosh \chi\end{array}\right)$, $\tanh 2 \chi=\frac{2 \sqrt{|\Lambda|}}{\mathcal{E}-\Lambda}$, and $\phi=e^{i \pi / 4}$ 
On the other hand, when $\Lambda>0$, the change of variable is $\left(\begin{array}{l}a_{+} \\ b_{+}\end{array}\right)=U\left(\begin{array}{l}\alpha_{+} \\ \beta_{+}\end{array}\right)$with $U=\left(\begin{array}{cc}\phi \cos \chi & \phi \sin \chi \\ -\bar{\phi} \sin \chi & \bar{\phi} \cos \chi\end{array}\right), \tan 2 \chi=\frac{2 \sqrt{\Lambda}}{\mathcal{E}-\Lambda}$.

\section{2 q-deformation with 2 degrees of freedom}

Deformation 1:

The most straightforward deformation is to deform the oscillators $\alpha, \beta$, at the stage where the hamiltonian is diagonal, in the form 4.1 .

One can choose to deform by a root of unity when $\Lambda>0$ :

$$
\begin{array}{ll}
\alpha_{-} \alpha_{+}-q \alpha_{+} \alpha_{-}=L_{\alpha}^{-1}, & L_{\alpha} \alpha_{ \pm} L_{\alpha}^{-1}=q^{ \pm 1} \alpha_{ \pm} \\
\beta_{-} \beta_{+}-q \beta_{+} \beta_{-}=L_{\beta}^{-1}, & L_{\beta} \beta_{ \pm} L_{\beta}^{-1}=q^{ \pm 1} \beta_{ \pm},
\end{array}
$$

with $[\alpha, \beta]=0$, and pick a hamiltonian, for example we can take $H_{B}$ from section 2.3 to get a supersymmetric model:

$$
H=\frac{\omega_{+}}{2}\left|\beta_{+} \beta_{-}+\beta_{-} \beta_{+}\right|+\frac{\omega_{-}}{2}\left|\alpha_{+} \alpha_{-}+\alpha_{-} \alpha_{+}\right|,
$$

while one can deform by $q$ real when $\Lambda<0$ :

$$
\begin{array}{cl}
\alpha_{-} \alpha_{+}-q \alpha_{+} \alpha_{-}=q^{-N_{\alpha}}, & {\left[N_{\alpha}, \alpha_{ \pm}\right]= \pm \alpha_{ \pm}} \\
\beta_{+} \beta_{-}-q \beta_{-} \beta_{+}=q^{-N_{\beta}}, & {\left[N_{\beta}, \beta_{ \pm}\right]=\mp \beta_{ \pm},}
\end{array}
$$

while again $[\alpha, \beta]=0$ and note that $\beta_{+} \leftrightarrow \beta_{-}$have exchanged roles. One could imagine a formula such as $q=e^{i \sqrt{\Lambda}}$, which requires $\Lambda$ to be quantized if it is positive [6], while if $\Lambda$ is negative, $q$ is real. This deformation removes the requirement of fixing the value of the angular momentum $J$ to get a finite number of states for $\Lambda>0$.

Deformation 2:

We could deform the model at an earlier stage, when the hamiltonian is not yet diagonal, but the commutation relations are canonical ${ }^{10}$. When the hamiltonian takes the form 4.3, we can deform the oscillators $a$ and $b$. Depending on the sign of $\Lambda$, we would use

$$
\begin{aligned}
& a_{-} a_{+}-q a_{+} a_{-}=L_{a}^{-1}, \quad L_{a} a_{ \pm} L_{a}^{-1}=q^{ \pm 1} a_{ \pm} \\
& b_{-} b_{+}-q b_{+} b_{-}=L_{b}^{-1}, \quad L_{b} b_{ \pm} L_{b}^{-1}=q^{ \pm 1} b_{ \pm} \text {, }
\end{aligned}
$$

\footnotetext{
${ }^{10}$ We do not know how to deform the model when the commutation relations are not canonical.
} 
for $\Lambda>0$, with $[a, b]=0$ or

$$
\begin{aligned}
a_{-} a_{+}-q a_{+} a_{-}=q^{-N_{a}}, & {\left[N_{a}, a_{ \pm}\right]= \pm a_{ \pm} } \\
b_{+} b_{-}-q b_{-} b_{+}=q^{-N_{b}}, & {\left[N_{b}, b_{ \pm}\right]=\mp b_{ \pm}, }
\end{aligned}
$$

with $\Lambda<0$ and again $[a, b]=0$.

Since the hamiltonian is no longer diagonalizable exactly, we studied this deformation with Mathematica. We include a sample of the code in an appendix, to illustrate the straightforwardness of doing such numerical computations. In Deformation 1, for $\omega_{+} \gg \omega_{-}$, the spectrum exhibits clear Landau levels. The simulation indicates that Landau level features are still somewhat present for Deformation 2.

\subsection{Noncommutative SUSY quantum mechanics with a magnetic field}

We have seen previously that the model of BNS features a non-analyticity in $\Lambda$ : the hamiltonian depends on $|\Lambda|$, the harmonic oscillator frequencies $\omega_{ \pm}$feature an absolute value, and the spectrum is qualitatively different depending on the sign of $\Lambda$. We thus decided to supersymmetrize this model to see if it would feature some enhancement of supersymmetry breaking as desired in a cosmological supersymmetry breaking scenario. We would need for that to find a quantity that depends on the derivative of $\Lambda$. The derivatives of the energy are discontinuous. For example, the ground state energy:

$$
\left.\frac{\partial E}{\partial \Lambda}\right|_{\Lambda \geq 0}=1 \quad \text { but }\left.\quad \frac{\partial E}{\partial \Lambda}\right|_{\Lambda \leq 0}=\frac{1-\theta^{2} \omega^{2}}{1+\theta^{2} \omega^{2}} \rightarrow-1 \text { for large } \theta \omega .
$$

There are two bosonic degrees of freedom $X_{i}, P_{i}, i=1,2$, and four real fermions $\psi_{\alpha}$, $\alpha=1, \ldots, 4$. They satisfy the commutation relations:

$$
\left[X_{1}, X_{2}\right]=i \theta, \quad\left[X_{i}, P_{j}\right]=i \delta_{i j}, \quad\left[P_{1}, P_{2}\right]=i B, \quad \text { and } \quad\left\{\psi_{\alpha}, \psi_{\beta}\right\}=\delta_{\alpha \beta}
$$

It is more convenient to work with complex/light-cone coordinates:

$$
X_{ \pm}=\frac{X_{1} \mp i X_{2}}{\sqrt{2}}, \quad P_{ \pm}=\frac{P_{1} \mp i P_{2}}{\sqrt{2}}, \quad \psi_{ \pm}=\frac{\psi_{1} \pm i \psi_{2}}{\sqrt{2}}, \quad \text { and } \quad \chi_{ \pm}=\frac{\psi_{3} \pm i \psi_{4}}{\sqrt{2}}
$$

The commutators are now:

$$
\left[X_{-}, X_{+}\right]=\theta, \quad\left[X_{ \pm}, P_{\mp}\right]=i, \quad\left[P_{-}, P_{+}\right]=B, \quad\left\{\psi_{+}, \psi_{-}\right\}=1, \quad \text { and } \quad\left\{\chi_{+}, \chi_{-}\right\}=1
$$

There are two supercharges:

$$
Q_{+}=\sqrt{2}\left(P_{-} \psi_{+}+W\left(X_{-}\right) \chi_{+}\right), \quad \text { and } \quad Q_{-}=\sqrt{2}\left(P_{+} \psi_{-}+\bar{W}\left(X_{+}\right) \chi_{-}\right)
$$


from which we get the hamiltonian:

$$
\begin{aligned}
& H=\frac{1}{2}\left\{Q_{+}, Q_{-}\right\}=\left(P_{+} P_{-}+\bar{W}\left(X_{+}\right) W\left(X_{-}\right)\right)+ \\
& \quad\left(\theta W^{\prime}\left(X_{-}\right) \bar{W}^{\prime}\left(X_{+}\right) \chi_{+} \chi_{-}+B \psi_{+} \psi_{-}+i W^{\prime}\left(X_{-}\right) \chi_{+} \psi_{-}-i \bar{W}^{\prime}\left(X_{+}\right) \psi_{+} \chi_{-}\right) .
\end{aligned}
$$

¿From now on, we will specialize to the case of a linear superpotential $W\left(X_{-}\right)=\omega X_{-}$, and then the hamiltonian is free:

$$
H=P_{+} P_{-}+\omega^{2} X_{+} X_{-}+\theta \omega^{2} \chi_{+} \chi_{-}+B \psi_{+} \psi_{-}+i \omega \chi_{+} \psi_{-}-i \omega \psi_{+} \chi_{-} .
$$

The bosonic part being the same as for the BNS model, except for the shift in ground state energy, we will just quote the formulas for diagonalizing the fermions. The fermionic part of $H$ takes the form

$$
\left(\chi_{+} \psi_{+}\right)\left(\begin{array}{cc}
\theta \omega^{2} & i \omega \\
-i \omega & B
\end{array}\right)\left(\begin{array}{l}
\chi_{-} \\
\psi_{-}
\end{array}\right) .
$$

Its eigenvalues are $\omega_{ \pm}$. The eigenvectors are, for $\Lambda>0$ :

$$
v_{ \pm}=\left(\left(B-\theta \omega^{2}\right)^{2}+4 \omega^{2}\right)^{-1 / 4}\left(\begin{array}{c}
i\left(\omega_{ \pm}+B\right)^{1 / 2} \\
\omega\left(\omega_{ \pm}+B\right)^{-1 / 2}
\end{array}\right) .
$$

After diagonalization, the hamiltonian takes the form:

$$
H=\omega_{+}\left(n_{+}+\frac{1}{2} \pm \frac{1}{2}\right)+\omega_{-}\left(n_{-}+\frac{1}{2} \pm \frac{1}{2}\right),
$$

with the frequencies given by the same expression 4.2 as in the BNS model.

Next, one can $q$-deform the model, along Deformation 1 or 2 above for example, and analyze the spectrum. Again, we find supersymmetry breaking by a small amount, qualitatively similar to the results presented in Table 1.

\section{Conclusion}

To get a parametrically large breaking of supersymmetry, one would hope that if some of the matrix elements in the hamiltonian differ by some small amount of order $\epsilon$, then the splitting in eigenvalues is enhanced to order $\epsilon^{\alpha}$ for $\alpha \in(0,1)$. For example, this would arise for the hamiltonian $\left(\begin{array}{c}1+\epsilon-1 \\ 1\end{array}-1\right)$, for which the eigenvalue splitting is of order $\sqrt{\epsilon}$. However this hamiltonian is not hermitian. On a highly speculative note, it would be very interesting if unitarity violation was the cause of supersymmetry breaking, and of the cosmological constant. 


\section{Acknowledgments}

We would like to thank Willy Fischler for suggesting this project, and for encouragements and discussions at all stages of this project. We would also like to acknowledge useful correspondence with V. Nair, A. Nersessian, M. Plyushchay, Y. Saint-Aubin and C. Sochichiu and thank S. Bellucci, D. Freed, J. Distler, S. Majid, E. Nicholson, S. Paban and L. Smolin for comments, criticisms or discussions. This work was supported in part by NSF grant PHY-0071512 and in part by a PPARC post-doctoral grant.

\section{A. Mathematica Code}

This very simple code computes the spectrum of the hamiltonian of section 4.2 . It should be easy to see how to adapt it to perform the other computations presented in this article.

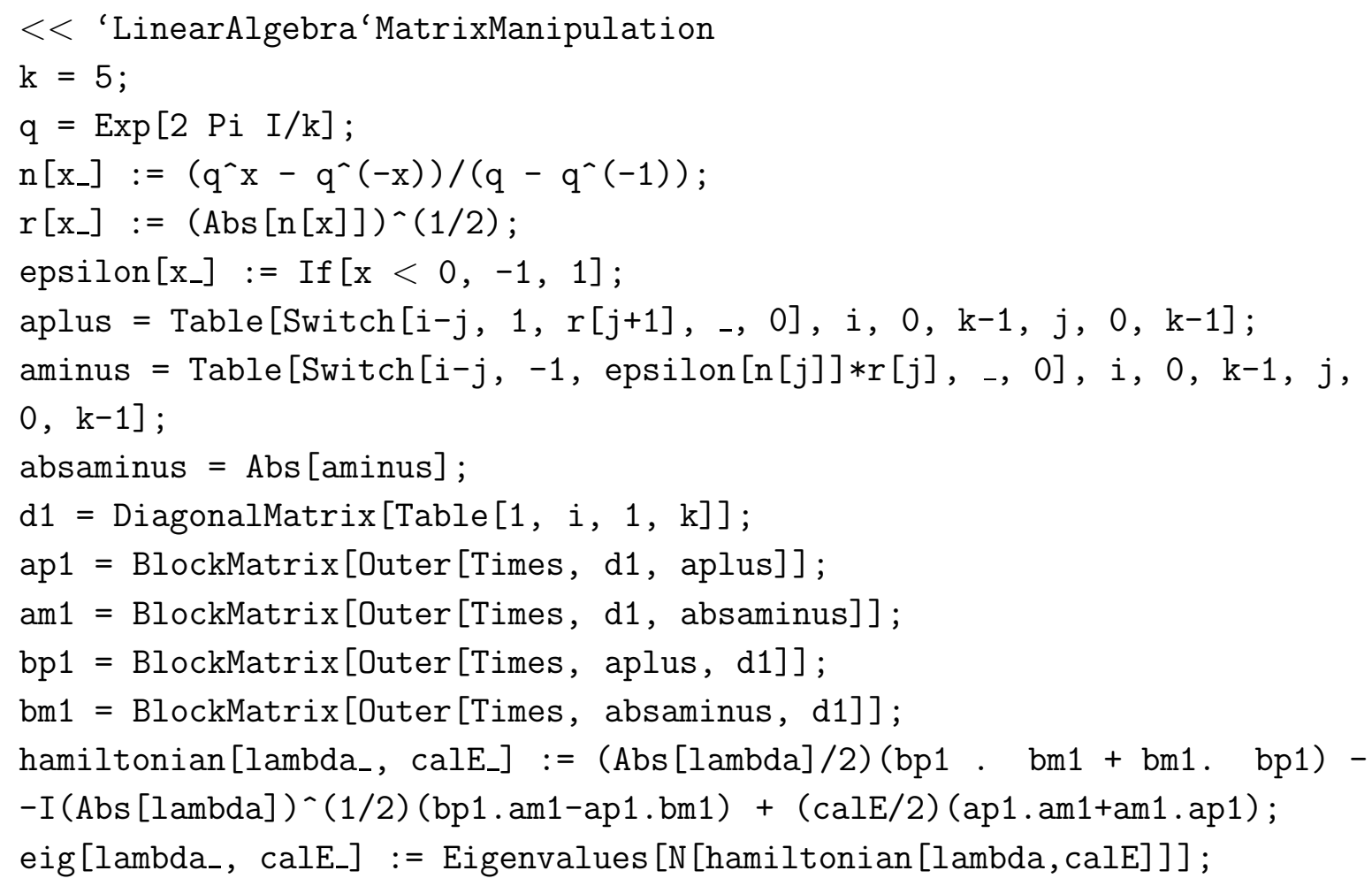




\section{References}

[1] G. 't Hooft, "Dimensional Reduction In Quantum Gravity," arXiv:gr-qc/9310026.

C. B. Thorn, "Reformulating string theory with the $1 / \mathrm{N}$ expansion," arXiv:hep-th/9405069.

L. Susskind, "The World as a hologram," J. Math. Phys. 36, 6377 (1995) [arXiv:hep-th/9409089].

[2] W. Fischler, "Taking de Sitter Seriously", Talk given at The Role of Scaling Laws in Physics and Biology (Celebrating the 60th Birthday of Geoffrey West), Santa Fe Dec. 2000 .

[3] T. Banks, "Cosmological breaking of supersymmetry or little Lambda goes back to the future. II," arXiv:hep-th/0007146.

T. Banks, "SUSY Breaking, Cosmology, Vacuum Selection and the Cosmological Constant in String Theory," arXiv:hep-th/9601151.

[4] R. Bousso, "Positive vacuum energy and the N-bound," JHEP 0011, 038 (2000) [arXiv:hep-th/0010252].

[5] S. Majid, "Hopf Algebras For Physics At The Planck Scale," Class. Quant. Grav. 5, 1587 (1988).

[6] S. Major and L. Smolin, "Quantum deformation of quantum gravity," Nucl. Phys. B 473, 267 (1996) [arXiv:gr-qc/9512020].

[7] L. Crane, "Categorical Physics," arXiv:hep-th/9301061.

L. Crane, "Clock and category: Is quantum gravity algebraic?," J. Math. Phys. 36, 6180 (1995) [arXiv:gr-qc/9504038].

L. Smolin, "Linking topological quantum field theory and nonperturbative quantum gravity," J. Math. Phys. 36, 6417 (1995) [arXiv:gr-qc/9505028].

[8] L. Smolin, "Quantum gravity with a positive cosmological constant," arXiv:hep-th/0209079.

[9] L. C. Biedenharn and M. A. Lohe, "Quantum Group Symmetry And Q Tensor Algebras," Singapore, Singapore: World Scientific (1995) 293 p.

[10] L. C. Biedenharn, "The Quantum Group SU(2)-Q And A Q Analog Of The Boson Operators," J. Phys. A 22, L873 (1989).

A. J. Macfarlane, "On Q Analogs Of The Quantum Harmonic Oscillator And The Quantum Group SU(2)-Q,” J. Phys. A 22, 4581 (1989). 
T. Hayashi, "Q Analogs Of Clifford And Weyl Algebras: Spinor And Oscillator Reprsentations Of Quantum Enveloping Algebras," Commun. Math. Phys. 127, 129 (1990).

[11] J. H. Petersen, "Representations at a root of unity of q oscillators and quantum Kac-Moody algebras," hep-th/9409079.

[12] I. M. Burban and A. U. Klimyk, "On Spectral Properties of q-Oscillator Operators", Lett. Math. Phys. 29,13 (1993).

[13] G. Mack and V. Schomerus, "Action of truncated quantum groups on quasiquantum planes and a quasiassociative differential geometry and calculus," Commun. Math. Phys. 149, 513 (1992).

[14] D. Arnaudon, "Composition of kinetic momenta: The U-q (sl(2) case," Commun. Math. Phys. 159, 175 (1994) [arXiv:hep-th/9212067].

[15] E. Witten, Nucl. Phys. B 188, 513 (1981).

[16] V. P. Nair and A. P. Polychronakos, "Quantum mechanics on the noncommutative plane and sphere," Phys. Lett. B 505, 267 (2001) [hep-th/0011172].

[17] S. Bellucci, A. Nersessian and C. Sochichiu, "Two phases of the non-commutative quantum mechanics," hep-th/0106138. 